\title{
A NEW DEFINITION OF THE GENERAL ABELIAN LINEAR GROUP*
}

\author{
BY
}

\section{LEONARD EUGENE DICKSON}

1. WE may give a striking definition of the general Abelian group, making use of the fruitful conception of the "compounds of a given linear homogeneous group," introduced in recent papers by the writer. $\dagger$ In $\S 3$ we determine the multiplicity of the isomorphism of a given linear homogeneous group to its compound groups. This result is applied in $\S 4$ to show the simple relation of the Abelian group to the general linear homogeneous group in the same number of variables. In $\S 5$ it is shown that the simple groups of composite order which are derived from the decompositions of the quaternary Abelian group and the quinary orthogonal group, each in the $G F\left[p^{n}\right], p>2$, are simply isomorphic. The investigation affords a proof of the simple isomorphism between the corresponding ten-parameter projective groups without the consideration of their infinitesimal transformations.

2. It will be convenient to introduce a notation more compact than that usually employed $\ddagger$ for the substitutions of the general Abelian group $A_{2 m, p^{n}}$ on $2 m$

\footnotetext{
* Presented to the Society at the Columbus meeting, A ugust 26, 1899, and in abstract at the meeting of February, 1899, under the title, Concerning the Abelian and hypoabelian groups. Received for publication August 26, 1899.

† Concerning a linear homogeneous group in $C_{m, q}$ variables isomorphic to the general linear homogeneous group in $m$ variables, Bull eti $n$, Dec., 1898.

The structure of certain linear groups with quadratic invariants, Proceedings of the London Mathematical Society, vol. 30, pp. 70-98.

‡Dickson, The Structure of the Hypoabelian Groups, Bulletin, pp. 495-510, July, 1898 ; A Triply Infinite System of Simple Groups, The Quarterly Journal of Pure and A pplied Mathematics, pp. 169-178, 1897 ; JoRDAN, Traité des Substitutions, pp. 171-179, for the case $n=1$.

Instead of considering the cogredient linear substitutions leaving invariant (up to a factor $a$ ) the usual bilinear function it is convenient to consider here the substitutions $A$ leaving invariant the function

$$
\psi \equiv \sum_{j=1}^{m}\left|\begin{array}{ll}
\xi_{2 j-1} & \xi_{2 j} \\
\xi_{2 j-1}^{n} & \xi_{2 j}^{p^{n}}
\end{array}\right|
$$
}

The conditions that $A$ shall leave $\psi$ invariant are seen to be (1). The hyperabelian group of linear homogeneous substitutions in the $G F\left[p^{2 n}\right]$ on $2 m$ indices which leave $\psi$ invariant has been studied by the writer in an article presented to the London Mathematical Society. 
indices in the Galois field of order $p^{n}$. The conditions that a substitution

$$
A: \quad \xi_{i}^{\prime}=\sum_{j=1}^{2 m} \alpha_{i j} \xi_{j} \quad(i=1, \cdots, 2 m)
$$

shall be Abelian are the following:

$$
\sum_{j=1}^{m}\left|\begin{array}{ll}
\alpha_{2 j-1 i} & \alpha_{2 j-1 k} \\
\alpha_{2 j i} & \alpha_{2 j k}
\end{array}\right|=\alpha \varepsilon_{i k} \quad(i, k=1, \cdots, 2 m ; i<k)
$$

where $\alpha$ is a parameter $\neq 0$ depending upon the particular substitution $A$ and where every $\varepsilon_{i k}=0$ unless $k=i+1=$ even, when

$$
\varepsilon_{2 l-12 l}=1
$$$$
(l=1, \cdots, m) .
$$

The second compound $C_{2 m, 2}$ of the $2 m$-ary group $A_{2 m, p^{n}}$ is formed by the substitutions

$$
Y_{i_{1} i_{2}}^{\prime}=\sum_{j_{1}<j_{2}}^{j_{1}, j_{2}=1 \ldots 2 m}\left|\begin{array}{ll}
\alpha_{i_{1} j_{1}} & \alpha_{i_{1} j_{2}} \\
\alpha_{i_{2} j_{1}} & \alpha_{i_{2} j_{2}}
\end{array}\right| Y_{j_{1} j_{2}} \quad\left(\begin{array}{c}
i_{1}, i_{2}=1, \ldots, 2 m \\
i_{1}<i_{2}
\end{array}\right) .
$$

We readily verify that the group $C_{2 m, 2}$ has the relative invariant

$$
Z \equiv \sum_{l=1}^{m} Y_{2 l-12 l}
$$

Indeed, in virtue of the relations (1), we have, on applying to $Z$ the substitution (2),

$$
\begin{gathered}
\sum_{l=1}^{m} Y_{2 l-12 l}^{\prime}=\sum_{j_{1}, j_{2}}\left\{\sum_{l=1}^{m}\left|\begin{array}{ll}
\alpha_{2 l-1 j_{1}} & \alpha_{2 l-1 j_{2}} \\
\alpha_{2 l j_{1}} & \alpha_{2 l j_{2}}
\end{array}\right|\right\} Y_{j_{1} j_{2}} \\
=\alpha \sum_{j_{1}, j_{2}} \varepsilon_{j_{1} j_{2}} Y_{j_{1} j_{2}}=\alpha \sum_{l=1}^{m} Y_{2 l-12 l} .
\end{gathered}
$$

Inversely, if the substitution (2) multiply the function $Z$ by a constant $\alpha$, the relations (1) hold true. We have proved the result:

Theorem.-The general Abelian group $A_{2 m, p^{n}}$ is the largest 2m-ary linear homogeneous group whose second compound has as a relative invariant the linear function $Z$.

3. To establish the more important theorem of $\S 4$, we determine the multiplicity of the isomorphism of a given $m$-ary linear homogeneous group $G_{m}$ to its $q^{\text {th }}$ compound $C_{m, q}$, supposing that $q<m$. To the substitution

$$
\left(\alpha_{i j}\right) \quad(i, j=1, \cdots, m)
$$

of $G_{m}$ there corresponds the following substitution of $C_{m q}: *$

\footnotetext{
* We employ Sylvester's umbral notation for determinants.
} 
$[\alpha]_{q}: \quad Y_{i_{1} \cdots i_{q}}^{\prime}=\sum_{\substack{j_{1} \ldots j_{q} \\ j_{1}<j_{2} \cdots j_{q}}}^{1, \ldots m}\left|\begin{array}{l}i_{1} \cdots i_{q} \\ j_{1} \cdots j_{q}\end{array}\right| Y_{j_{1} \cdots j_{q}} \quad\left(\begin{array}{c}i_{1}, \cdots, i_{q}=1, \cdots, m \\ i_{1}<i_{2}<\cdots<i_{q}\end{array}\right)$.

Let $j$ be an integer such that $q<j \equiv m$. Consider the matrix $J$ of certain coefficients of the substitution $[\alpha]_{q}$, viz.,

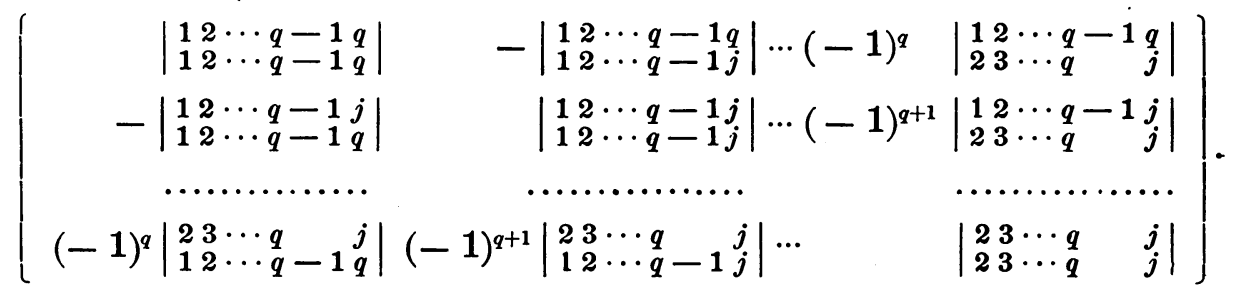

Consider also the matrix $A$ of determinant $\Delta$,

$$
A \equiv\left(\begin{array}{cccc}
\alpha_{j j} & \alpha_{q j} & \cdots & \alpha_{1 j} \\
\alpha_{j q} & \alpha_{q q} & \cdots & \alpha_{1 q} \\
\cdots \cdots & \cdots & \cdots & \cdots \\
\alpha_{j 1} & \alpha_{q 1} & \cdots & \alpha_{11}
\end{array}\right] .
$$

The composition of the matrices $J$ and $A$ gives the result

$$
J A \equiv\left(\begin{array}{cccc}
\Delta & 0 & \ldots & 0 \\
0 & \Delta & \ldots & 0 \\
\ldots & \ldots & \ldots & . \\
0 & 0 & \ldots & \Delta
\end{array}\right]
$$

We seek those substitutions of $G_{m}$ which correspond to the identity in $C_{n, q^{-}}$ Suppose, therefore, that $[\alpha]_{q}$ reduces to the identical substitution, so that the matrix $J$ is the identity.

In this case we have

$$
\Delta^{q+1}=\Delta, \alpha_{i k}=0, \alpha_{i i}=\Delta \quad(i, k=1,2 \cdots, q, j ; i \neq k) .
$$

Taking in turn $j=q+1, q+2, \ldots, m$, we have the result

$$
\left(\alpha_{i j}\right) \equiv\left(\begin{array}{cccc}
\Delta & 0 & . & 0 \\
0 & \Delta & \ldots & 0 \\
\cdots & \ldots & \ldots \\
0 & 0 & . & \Delta
\end{array}\right) .
$$

Hence $\Delta \neq 0$ and therefore $\Delta^{q}=1$. Inversely, every such substitution of $G_{m}$ corresponds to the identity in $C_{m, q}$. 
Theorem.-The continuous group of all m-ary linear homogeneous substitutions is $(q, 1)$ fold isomorphic to its $q^{\text {th }}$ compound $(q<m)$.

For linear substitutions in the $G F\left[p^{n}\right]$, we have

$$
\Delta^{q}=1, \quad \Delta^{p^{n}-1}=1 .
$$

Thus we have the analogous.

Theorem.-The group of all m-ary linear homogeneous substitutions in the $G F\left[p^{n}\right]$ is $(g, 1)$ fold isomorphic to its $q^{\text {th }}$ compound, $g$ being the greatest common divisor of $q$ and $p^{n}-1$.

4. From the results of $\S \S 2-3$, we derive immediately the

Theorem.-According as $p=2$ or $p>2$, the general Abelian group $A_{2 m, p^{n}}$ is holoedrically or hemiedrically* isomorphic to that subgroup of the second compound of the general $2 m$-ary linear homogeneous group in the $G F\left[p^{n}\right]$ which has as a relative invariant the linear function $Z$.

The writer has shown ( $\mathrm{Bulletin,l.c.)}$ that this second compound leaves invariant the Pfaffian

$$
F_{2 m} \equiv[1,2, \cdots, 2 m]
$$

Hence $A_{2 m, p^{n}}$ is isomorphic to a linear homogeneous group in $m(2 m-1)$ variables $Y_{i j}$ with coefficients in the $G F\left[p^{n}\right]$ and having as relative invariants the functions

$$
F_{2 m}, Z \equiv \sum_{l=1}^{m} Y_{2 l-12 l}
$$

To the subgroup* of the latter which leaves these functions absolutely invariant there corresponds a self-conjugate subgroup of $A_{2 m} p^{n}$, which leaves the customary bilinear function absolutely invariant. This subgroup, containing only substitutions of determinant \pm 1 , may be designated as the special Abelian group $A_{2 m \cdot \eta^{n}}^{\prime} \quad$ It has the self-conjugate substitution which changes the signs of all the $2 m$ indices. Except for $\left(2 m, p^{n}\right)=(2,2),(2,3)$ and $(4,2)$, the quotient group $h_{2 m, p^{n}}$ is simple.t

5. THEOREM.-For $p>2$, the simple group $H_{4, p^{n}}$, having the order

* Since the Abelian group contains the substitution $\xi_{i}=-\xi_{i}(i=1,2, \ldots, 2 m)$.

$\dagger$ This group has been studied by the writer in the Proceedings of the London Mathematical Society, l. c., $8 z 22-33$.

$\ddagger$ Dickson, A triply infinite system of simple groups, The Quarterly Journal of Mathematics, July, 1897 ; ibid., April, 1899, for the cases $p=2,2 m=4, n>1$, previously unconsidered. 


$$
\frac{1}{2}\left(p^{4 n}-1\right)\left(p^{2 n}-1\right) p^{4 n}
$$

is simply isomorphic to the simple subgroup of equal order of the quinary orthogonal group in the $G F^{\prime}\left[p^{n}\right]$.

On introducing the invariant $Z \equiv Y_{12}+Y_{34}$ as a new variable in place of $Y_{34}$, the general substitution [see (2)] of the second compound of $A_{4, p^{n}}^{\prime}$ becomes, for $p>2$ :

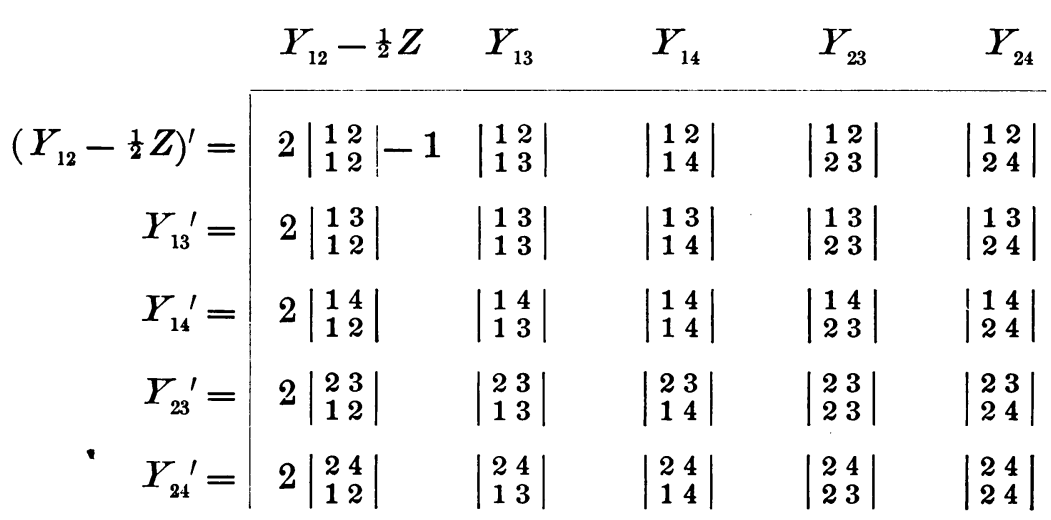

It is therefore a substitution on five indices leaving absolutely invariant the function

$$
\varphi \equiv\left(\frac{1}{2} Z\right)^{2}-[1234] \equiv\left(Y_{12}-\frac{1}{2} Z\right)^{2}+Y_{13} Y_{24}-Y_{14} Y_{23}
$$

This second compound is simply isomorphic to $H_{4, p^{n}}$. Indeed, the former is hemiedrically isomorphic to $A_{4, p^{n}}^{\prime}$ by $\S 3$; while to the substitution changing the sign of every index there corresponds the identity in the second compound.

By a simple transformation of indices* the function $\varphi$ can be given the form

$$
\sum_{i=1}^{5} x_{i}^{2}
$$

Hence the second compound is simply isomorphic to a subgroup $O_{5, p^{n}}$ of the total orthogonal group $O$ of determinant unity. From the result of $\$ 16$ of the paper in the Proceedings of the London Mathematical Society, above cited, it follows that $O_{5, p^{n}}$ does not contain the substitution which extends the simple subgroup of $O$ of order

* See the first pages of the article, Determination of the structure of all linear homogeneous groups in a Galois field which are defined by a quadratic invariant, American Journal of Mathematics, July, 1899. 


$$
\frac{1}{2}\left(p^{4 n}-1\right)\left(p^{2 n}-1\right) p^{4 n}
$$

to the total group $O$. Hence $O_{5, p^{n}}$ is this simple subgroup.

This investigation also proves the theorem due to Lie: The projective group of a linear complex in space of three dimensions is isomorphic to the projective group of a non-degenerate surface of the second order in space of four dimensions, each group having ten parameters.

University of California, August 20, 1898. 